\title{
Consistency of observed near surface temperature trends with climate change projections over the Mediterranean region
}

\author{
Armineh Barkhordarian • Jonas Bhend • \\ Hans von Storch
}

Received: 24 November 2010/ Accepted: 24 March 2011/Published online: 7 April 2011

(C) The Author(s) 2011. This article is published with open access at Springerlink.com

\begin{abstract}
We examine the possibility that anthropogenic forcing (Greenhouse gases and Sulfate aerosols, GS) is a plausible explanation for the observed near-surface temperature trends over the Mediterranean area. For this purpose, we compare annual and seasonal observed trends in near-surface temperature over the period from 1980 to 2009 with the response to GS forcing estimated from 23 models derived from CMIP3 database. We find that there is less than a $5 \%$ chance that natural (internal) variability is responsible for the observed annual and seasonal area-mean warming except in winter. Using additionally two pattern similarity statistics, pattern correlation and regression, we find that the large-scale component (spatial-mean) of the GS signal is detectable (at $2.5 \%$ level) in all seasons except in winter. In contrast, we fail to detect the small-scale component (spatial anomalies about the mean) of GS signal in observed trend patterns. Further, we find that the recent trends are significantly (at $2.5 \%$ level) consistent with all the 23 GS patterns, except in summer and spring, when 9 and 5 models respectively underestimate the observed warming. Thus, we
\end{abstract}

Electronic supplementary material The online version of this article (doi:10.1007/s00382-011-1060-y) contains supplementary material, which is available to authorized users.

A. Barkhordarian $(\bowtie) \cdot J$. Bhend $\cdot H$. von Storch

Institute for Coastal Research, Helmholtz-Zentrum Geesthacht,

Geesthacht, Germany

e-mail: armineh.barkhordarian@hzg.de

Present Address:

J. Bhend

Centre for Australian Weather and Climate Research, CSIRO Marine and Atmospheric Research, Aspendale,

VIC, Australia

H. von Storch

KlimaCampus, Hamburg, Germany conclude that GS forcing is a plausible explanation for the observed warming in the Mediterranean region. Consistency of observed trends with climate change projections indicates that present trends may be understood of what will come more so in the future, allowing for a better communication of the societal challenges to meet in the future.

keywords Mediterranean - Detection .

Near-surface temperature

\section{Introduction}

The attribution of global temperature variations over the past century to a combination of anthropogenic and natural influences is now well established, with the anthropogenic factors dominating. However, difficulties remain in the detection of a human influence in observed trends at regional scales (Hegerl et al. 2007). This is a consequence of the increasing variability, and thus generally decreasing signal-to-noise ratio, with decreasing area of aggregation (Stott and Tett 1998; Zwiers and Zhang 2003). In addition, it is not always possible to account for all "external" factors, in particular when dealing with the regional scales of up to, thousand kilometres. On these scales, the effect of factors such as land use or land cover changes, emission of aerosols related to traffic, industry or natural sources may be very noisy (may depend on the circulation, which is related to internal variability) and any of which may be important contributors to the observed trends. Detection and attribution analyses must therefore be continuously updated as our understanding of the processes that govern climate change and variability accumulates.

The formal detection and attribution approach has been applied to study temperature changes over the southern 
Europe land area, which is a part of the Mediterranean region. External forcing on changes in area-average temperature has been detected and attributed to anthropogenic forcing over the European land area by Stott (2003) and by Stott et al. (2004). In addition, a detectable external influence on the spatiotemporal pattern of annual temperature anomalies has been found by Zhang et al. (2006). Christidis et al. (2010) use global constraints from a multimodel approach with three models and indicate that warming over the Mediterranean land area is likely due to anthropogenic influence.

In this study, we focus on the question whether the recent warming is a plausible harbinger of future warming - that is, we analyze whether the observed changes are consistent with climate change projections. By linking past changes to expected future changes, this analysis helps to provide an illustrative example of what a potential future climate influenced by enhanced greenhouse gas (GHG) concentrations might look like. When talking about the future, we are leaving the statistical area of quantifying the risk of incorrect assessments. Instead we are entering the field of plausibility. We link past and future changes using a set of hierarchical questions. First, we assess whether the observed recent changes are different from natural variability derived from the observed record with a bootstrap procedure. Second, we analyze whether the observed changes are consistent with GS (Greenhouse gases and Sulfate aerosols) forcing, taking into account that internal variability and other external forcing influence the observed record. Having established that external forcings are detectable and that GS forcing is a plausible explanation for the observed change, in the last step we assess whether the ensemble of projections encompass the observed warming-if this is the case, we conclude that the observed change can be interpreted as a harbinger of future change.

Consistency with projections-as defined above-does not demonstrate cause and effect relationships; these would require a formal attribution study (that we are unable to provide at the moment as we consider the understanding of many of the important forcing mechanisms at the regional scale as insufficient). Consistency, in contrast, points to the plausibility (not probability since this is a physical argument not a statistical argument) that the recent trend will continue into the future- based on the understanding that the recent trend is related to the known forcing, which will continue into the future. If we conclude that the observed change is consistent with climate change projections, our assessment provides an illustrative example of a potential future by comparing the observed change to one hypothetically dominant forcing (GS forcing in this case, see Bhend and von Storch (2008, 2009)).

We compare near-surface temperature trends over sea and land for the period from 1980 to 2009 with climate change projections derived from the set of global climate model simulations provided through the World Climate Research Programmes (WCRP) Coupled Model Intercomparison Project 3 (CMIP3, Meehl et al. (2007)). We analyze both annual and seasonal area-average change and pattern similarity. The method used in this study has not been applied to Mediterranean temperature before. Most studies in the Mediterranean region consider winter and summer regimes, while characterization of spring and autumn is more uncertain, revealing, presumably, the transient nature of these two seasons in the Mediterranean (Lionello et al. 1999). In contrast to other studies, we analyze the different seasons separately and present results for the 23 models individually.

The remainder of this paper is structured as follows. Details on the observational and model data are given in Sect. 2. The methodology used in this study is discussed in Sect. 3. The results including the detection, consistency with GS forcing, and the overall assessment whether the observed warming is consistent with the multitude of available projections are shown in Sect. 4 . The main conclusions and discussions are presented in Sect. 5.

\section{Observations and model data}

The Mediterranean area is defined as the region from $25^{\circ} \mathrm{N}$ to $50^{\circ} \mathrm{N}$ and $10^{\circ} \mathrm{W}$ to $40^{\circ} \mathrm{E}$ (see Fig. 1). Trends in observation data are computed from the HadCRUT3 dataset (Brohan et al. 2006). This dataset is based on a combination of monthly values of land near-surface air temperature anomalies and sea-surface temperature anomalies relative to $1961-1990$ and is presented on $5^{\circ}$ (latitude) by $5^{\circ}$ (longitude) grid for the period from 1850 to 2009 .

We use global simulations with 23 coupled AtmosphereOcean General Circulation Models (AOGCMs) to estimate the anthropogenic signal. The simulations are included in the World Climate Research Programme's (WCRP) Coupled Model Intercomparison Project 3 (CMIP3, Meehl et al. (2007)). A list of the climate models used in this study is given in Table 1 . The future projections are based upon the IPCC SRES A1B scenario with a $\mathrm{CO} 2$ concentration of $700 \mathrm{ppm}$ by the year 2100 .

\section{Methodology}

We first analyze whether external influences on the observed change are detectable. Therefore, we compare the observed change with estimates of the natural variability (i.e. internal variability and variability due to other unaccounted factors) derived from the observed record with a bootstrap procedure outlined in Sect. 3.3. We further 
Fig. 1 The left column Anthropogenic climate change signal in seasonal near-surface temperature according to CMIP3 multi-model ensemble mean with 23 models. The right column Observed seasonal trends over the time period from 1980 to 2009 derived from HadCRUT3 data
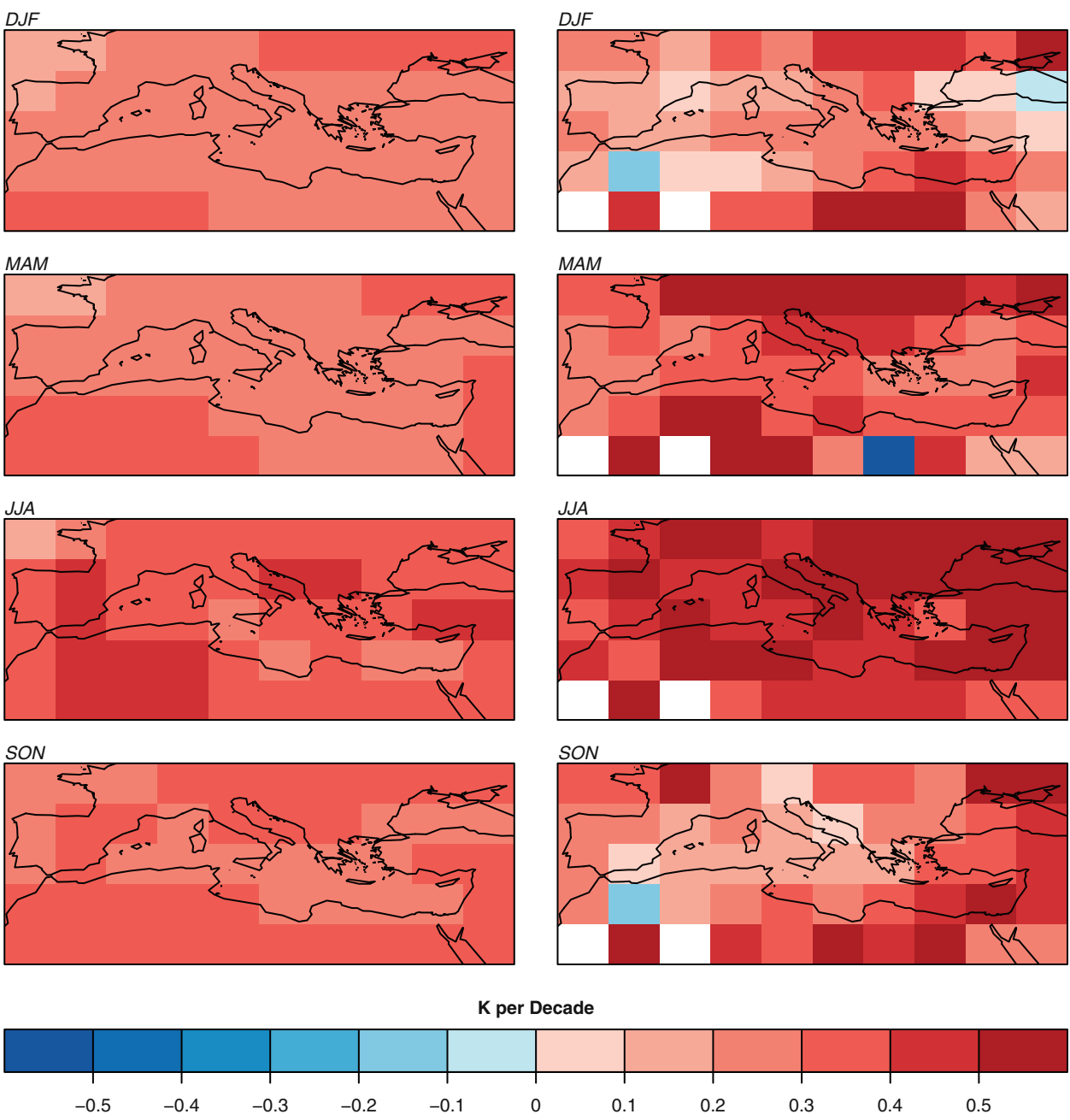

compare the observed change to estimates of internal variability derived from the control runs of the respective models. Second, we analyze if GHG and sulfate (GS) forcing is a plausible explanation for the observed change, taking into account, that both internal variability and other external (but unspecified) forcings influence the observed record. The analysis as described above is carried out for each of the models individually. The last step of the analysis, in contrast, is an overall assessment, we consider a large number of climate change projections according to the SRES A1B scenario, generated by 23 global models, we determine whether the recent trend is within this range of expected change due to GS forcing and could thus be seen as a harbinger of future change.

\subsection{Anthropogenic climate change signal estimates}

We define the anthropogenic climate change signal as the difference between the last decades of the twenty first century (2071-2100) and the reference climatology (1961-1990). We assume a linear development from 1961 to 2100 and the resulting signal is scaled to change per decade. Using well-separated time slices, 110 years in this study, has the advantage of increasing the signal-to-noise ratio and there is no need to average multiple models to get good signal estimates. Thus allowing us to investigate the robustness of our results to using different climate models and to explicitly deal with individual models separately.

Additional analyses show that by assuming a constant warming rate, we slightly overestimate the actual rate of warming from 1980 to 2009 (see Supplementary Fig. 1). We found no evidence of a discernible change in the pattern of warming during the period from 1961 to 2100 (see Supplementary Fig. 2). The advantage of a much higher signalto-noise ratio of an anthropogenic signal when estimated from time slices justifies the use of time-invariant warming patterns as opposed to transient warming patterns.

\subsection{Comparing the patterns of change}

The comparison of observed and anthropogenic climate change signal patterns are carried out using three pattern 
Table 1 Seasonal and annual un-centred pattern correlation coefficients of near-surface temperature for 30-year trends from 1980 to 2009 , compared to the trend of 23 anthropogenic climate change scenarios derived from the CMIP3 multi-model data set

\begin{tabular}{|c|c|c|c|c|c|c|}
\hline & Models & DJF & MAM & JJA & SON & Annua \\
\hline 1 & bccr-bcm2-0 & 8 & $*$ & $0.96^{*}$ & $0.87 *$ & $0.95^{*}$ \\
\hline 2 & cccma-cgcm3-1 & 7 & $*$ & 1* & 7* & $3 *$ \\
\hline 3 & cccma-cgem & 0.86 & $*$ & $2^{*}$ & & $0.93 *$ \\
\hline 4 & cnrm-cm3 & 0.88 & $0.87 *$ & $0.97 *$ & $8 *$ & $0.95^{*}$ \\
\hline 5 & csiro-mk3-0 & 0.82 & $0.87^{*}$ & $0.94 *$ & $0.89 *$ & $0.95^{*}$ \\
\hline 6 & csiro-mk3-5 & 0.89 & $0.84 *$ & $0.96^{*}$ & $0.89 *$ & $0.95^{*}$ \\
\hline 7 & gfdl-cm2-0 & 0.88 & $0.90^{*}$ & $0.96^{*}$ & $0.87 *$ & $0.95^{*}$ \\
\hline 8 & gfdl-cm2-1 & 0.89 & $0.87^{*}$ & $0.96^{*}$ & $0.87 *$ & $0.92 *$ \\
\hline 9 & giss-aom & 0.87 & $0.90^{*}$ & $0.94 *$ & $0.88^{*}$ & $0.94 *$ \\
\hline 10 & giss-model-e-h & 0.85 & $0.88^{*}$ & $0.94 *$ & $0.88^{*}$ & $0.95^{*}$ \\
\hline 11 & giss-model-e-r & 0.88 & $0.84 *$ & $0.92 *$ & $0.85^{*}$ & $0.92 *$ \\
\hline 12 & ingv-echam4 & 0.88 & $0.90^{*}$ & $0.94 *$ & 0. & $0.94 *$ \\
\hline 13 & inmem 3-0 & 0.87 & $0.87 *$ & $0.96^{*}$ & $0.89 *$ & $0.95^{*}$ \\
\hline 14 & ipsl-cm4 & 0.88 & $0.90^{*}$ & $0.96^{*}$ & $0.88^{*}$ & $0.95^{*}$ \\
\hline 15 & miroc3-2-hires & 0.88 & $0.88^{*}$ & $0.97 *$ & $0.88 *$ & $0.97 *$ \\
\hline 16 & miroc3-2-medre & 0.01 & $0.90 *$ & $0.96^{*}$ & $0.89 *$ & $0.96 *$ \\
\hline 17 & miub-echo-g & 0.89 & $0.93^{*}$ & $0.96^{*}$ & $0.87 *$ & $0.93 *$ \\
\hline 18 & mpi-echam5 & 0.88 & $0.88^{*}$ & $0.96^{*}$ & $0.88 *$ & $0.94 *$ \\
\hline 19 & mri-cgcm 2-3-2 & 0.87 & $0.91 *$ & $0.96^{*}$ & $0.87 *$ & $0.95^{*}$ \\
\hline 20 & ncar-ccsm3-0 & 0.89 & $0.92 *$ & $0.96^{*}$ & $0.88 *$ & $0.94 *$ \\
\hline 21 & ncar-pcm1 & 0.89 & $0.89 *$ & $0.94 *$ & $0.90 *$ & $0.96 *$ \\
\hline 22 & ukmo-hadem3 & 0.88 & $0.90 *$ & $0.97 *$ & $0.90 *$ & $0.95^{*}$ \\
\hline 23 & ukmo-hadgem1 & 0.87 & $0.92 *$ & $0.97 *$ & $0.89 *$ & $0.95 *$ \\
\hline
\end{tabular}

The indices significantly greater than zero at $2.5 \%$ level are labelled with an asterisk

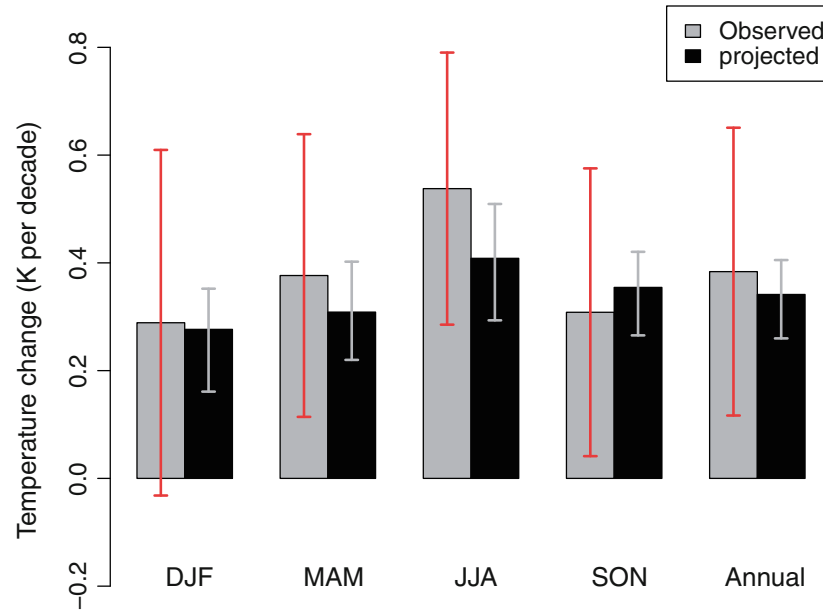

Fig. 2 Observed seasonal and annual area mean changes of near surface temperature over the period 1980 to 2009 in comparison with anthropogenic signals (GS) according to the SRES A1B scenario derived from the CMIP3 multi-model ensemble mean. The vertical axes shows area mean change of near surface temperature ( $\mathrm{K}$ per decade). The grey whiskers indicate the spread of trends of 23 climate change projections used in this study. The red whiskers denote the bootstrap $90 \%$ confidence interval of observed trends similarity statistics. We use both centred and un-centred pattern correlation (Eqs. 1 and 2). The un-centred correlation measures the similarity of two patterns without removal of the spatial mean, while the centred correlation refers to the correlation of deviation patterns, where the spatial mean has been subtracted (Santer et al. 1993). The third pattern similarity statistic is regression (Eq. 3). Unlike the correlation statistics, this measure includes information about the relative magnitudes of the observed and model projected trend patterns. Trends in observations have been calculated using ordinary least squares linear regression.

$$
\begin{aligned}
& U C(O, P)=\frac{\sum_{i=1}^{n} P_{i} \cdot O_{i}}{\sqrt{\sum_{i=1}^{n} P_{i}^{2} \cdot \sum_{i=1}^{n} O_{i}^{2}}} \\
& C C(O, P)=\frac{\sum_{i=1}^{n}\left(P_{i}-\bar{P}\right) \cdot\left(O_{i}-\bar{O}\right)}{\sqrt{\sum_{i=1}^{n}\left(P_{i}-\bar{P}\right)^{2} \cdot \sum_{i=1}^{n}\left(O_{i}-\bar{O}\right)^{2}}} \\
& R(O, P)=\frac{\sum_{i=1}^{n} P_{i} \cdot O_{i}}{\sum_{i=1}^{n} P_{i}^{2}}
\end{aligned}
$$

The index subscript $i=1, \ldots, n$ counts the spatial points. $O_{i}$ and $P_{i}$ refer to the observed and simulated pattern of change, respectively.

The un-centred correlation and regression statistics combine both spatial-mean and pattern information. In order to have a measure without the effect of spatial pattern information we also compare the area-mean changes of observed and anthropogenic signal patterns.

\subsection{Significance of pattern similarity statistics}

We use a bootstrap technique to test the null hypothesis that the observed trends are drawn from an undisturbed stationary climate (von Storch and Zwiers 1999). Thus, we separate between GS-related change and non-GS variability. This non-GS variability includes all factors, which are assumed to remain stationary in the coming century, i.e. not only internal variability but also other unaccounted external factors, such as volcanoes, cosmic influences and aerosol forcing. We estimate non-GS variability by re-sampling the observational record using a moving block bootstrap technique (Wilks 1997).

The block length chosen for the moving blocks bootstrapping depends on the autocorrelation of the seasonal temperature time series. This is different for different grid boxes and different seasons. Our analysis based on a method suggested by Wilks (1997), indicates that the average block length across the Mediterranean is 2.2, 3.5, 5, and 2.8 for DJF, MAM, JJA and SON, respectively (see Supplementary Fig. 3). We choose a block length of 5 that should lead to slightly conservative confidence intervals at most grid boxes. We draw 1,000 30-year time series to 
Fig. 3 Seasonal pattern similarity statistics of the multimodel mean signal with observed moving 30-year trends. The vertical axes in a shows centered correlation coefficients in b Un-centered correlation coefficients and in c regression coefficients. The horizontal axes show the endyear of moving 30-year trends. The dotted horizontal lines indicate the $95 \%$ confidence interval derived from block bootstrapping (a)

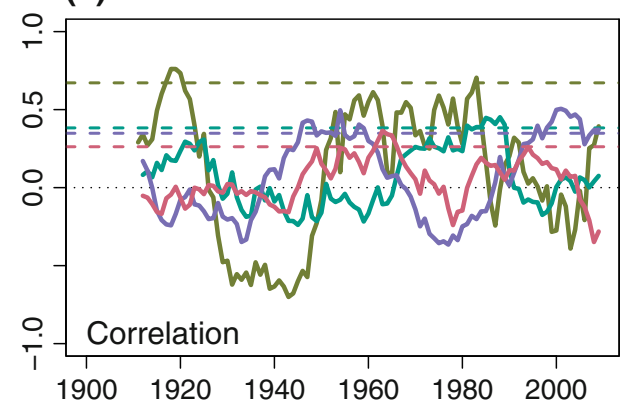

(c)

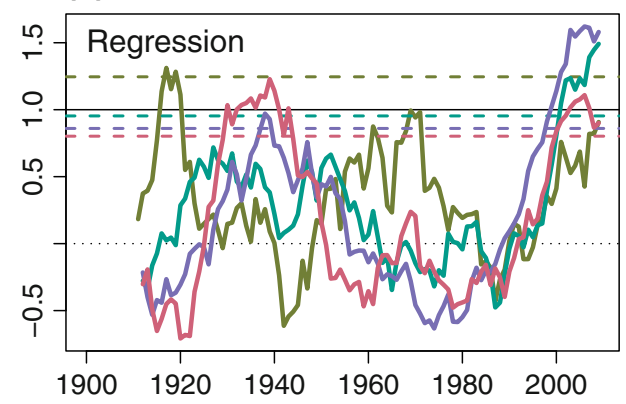

(b)

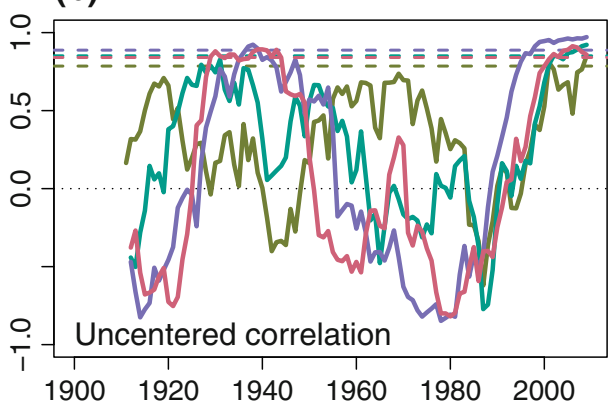

estimate the variability of 30-year trends in a stationary climate. Of course, question marks remain as to what extent the length of the observed record (160 years in this study) is sufficiently long for giving reliable estimates of variability.

Furthermore, we use the bootstrapped trend patterns to disturb the observed trend patterns and then compute the same pattern similarity statistics. By doing so, we sample the range of non-GS variability in the observed trends. Quantiles of these bootstrapped pattern similarity statistics are then used to describe the non-GS-variability of the pattern similarity statistics.

\section{Results}

4.1 Is the recently observed warming due to natural (internal) variability alone?

The comparison of observed area mean change of seasonal near-surface temperature over the period from 1980 to 2009 and the multi-model ensemble mean response (a mean over all available ensemble members) is shown in Fig. 2 . The observed warming is likely not due to natural variability (non-GS variability) alone in cases where the 90 percent uncertainty range (red bars in Fig. 2) derived from bootstrapped trends (Sect. 3.3) excludes zero. As shown in Fig. 2, externally forced changes are detected in the observed annual area-mean warming and in all seasons except winter.
To investigate the robustness of our results to using model-based internal variability, we compare the observationally based estimate of internal variability with the variability estimated from the control runs (climate model simulations in which all forcings are held constant), derived from the 23 models used in this study. Our results shows that in all seasons, the variability based on the control runs of the 23 models is smaller than the variability estimated from block bootstrapping (see Supplementary Fig. 4), indicating that the detection of externally forced changes in the observed trends over the Mediterranean is robust to using model-based estimates of internal variability.

\subsection{Is the effect of GS-forcing detectable} in the recently observed warming?

Table 1 shows the seasonal and annual un-centred pattern correlation coefficients of observed near-surface trends from 1980 to 2009 with anthropogenic signals derived from the 23 models in the CMIP3 archive. The annual un-centred correlation coefficients are in the range of 0.92 to 0.97 and these correlations are larger than the $95 \%$ quantiles of bootstrapped pattern correlations. In summer (JJA) all climate change projections share very high un-centred correlation coefficients ranging from 0.91 to 0.97 which are significant at the $2.5 \%$ level. The correlation of observed trend patterns with anthropogenic signal patterns is also high in spring (MAM) and autumn (SON). In spring, the coefficients are ranging from 0.87 to 0.93 (significant at 
Fig. 4 Regression coefficients ( $y$ axes) of observed nearsurface temperature changes against simulated in response to GS forcing according to the SRES A1B scenario derived from 23 models ( $x$ axes). The bars show the $95 \%$ uncertainty ranges of regression coefficients derived from the observed record using a moving blocks bootstrap. The solid horizontal lines mark regression indices equal to unit scaling indicating consistency with GS forcing
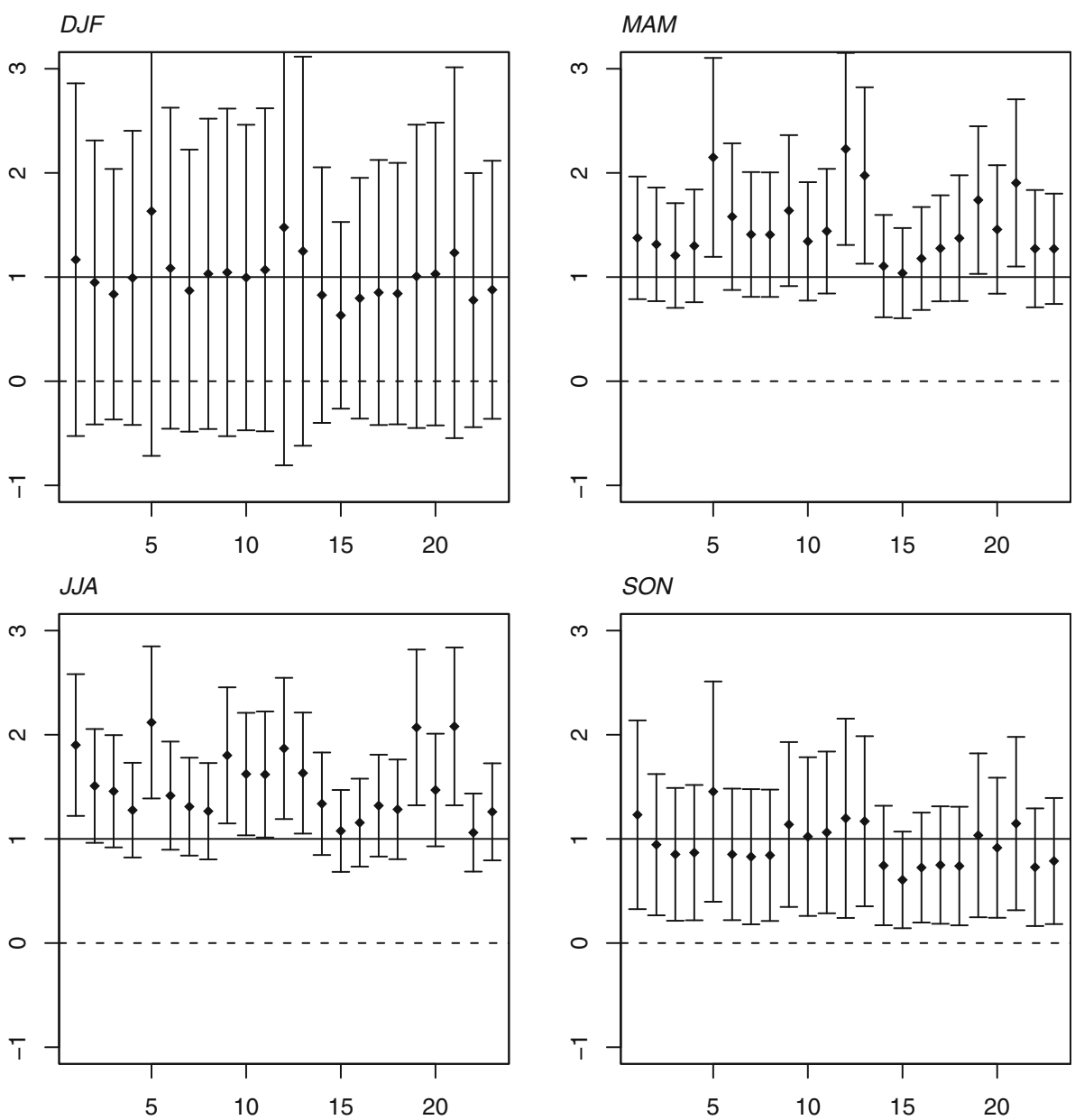

$2.5 \%$ level) and in autumn from 0.85 to 0.90 (significant at $2.5 \%$ level). Indeed such correspondence can hardly (less than $2.5 \%$ risk level) be expected to show up if only nonGS forcing would be present. Although, we do not find a detectable external influence using centred pattern correlation, in which the spatial-mean is removed and the pattern is simply a spatial anomaly pattern(not shown).

When using regression as a pattern similarity measure, which unlike the correlation statistics measures the relative magnitudes of the observed and model projected trend patterns, we are able to detect external influences in annual warming and in all seasons except in winter. Figure 3 displays the regression coefficients and its $95 \%$ confidence interval. Detection of GS signal is claimed at $2.5 \%$ significant level when the uncertainty range does not include zero. The regression coefficients with individual models and their significance levels are presented in Table 2. As shown in Fig. 3 in spring, summer and autumn the uncertainty interval does not include the zero line in all cases. Therefore, we conclude that there is less than a $2.5 \%$ chance that natural (internal) variability rather than the GS signal is responsible for the observed change.
Significant un-centred correlation coefficients and regression indices clearly indicate that the combined largescale (spatial mean) and small-scale (anomalies about the mean) component of GS signal is detected in annual mean warming and all seasons except in winter. Failure to detect the smaller-scale component (spatial anomalies about the mean) of GS signal in observed trend patterns indicates that the spatial-mean is the important and dominant component of the GS signal. We have to be aware, however, that both spatial coverage and representativeness of the observations as well as potential model errors at grid-box-scale have a strong influence on the similarity of spatial anomaly patterns.

We further analyze how strongly our results depend on the exact time period under analysis. Figure 3 shows the pattern similartiy statistics of the multi-model mean signal with moving 30-year trends in the HadCRUT3 dataset. We find that the centred correlation (Fig. 3a) is with a few exceptions never significantly different from zero. The uncentred correlation and regression (except in winter), however, becomes significant for 30-year trends ending in 2000 and later. The regression (Fig. 3b) and uncentred 
Table 2 Seasonal and annual regression coefficients of near-surface temperature for 30-year trends from 1980 to 2009, compared to the trend of 23 anthropogenic climate change scenarios derived from the CMIP3 multi-model dataset

\begin{tabular}{|c|c|c|c|c|c|c|}
\hline & Models & DJF & MAM & JJA & SON & Annual \\
\hline 1 & bccr-bcm2-0 & 1.1 & $1.3^{*}$ & $1.9^{*}$ & $1.2 *$ & $1.1^{*}$ \\
\hline 2 & cccma-cgem3-1 & 0.94 & $1.3^{*}$ & $1.5 *$ & $0.94 *$ & $0.92 *$ \\
\hline 3 & cccma-cgem3-1-t63 & 0.83 & $1.2^{*}$ & $1.4^{*}$ & $0.85 *$ & $0.84 *$ \\
\hline 4 & $\mathrm{cnrm}-\mathrm{cm} 3$ & 0.99 & $1.2 *$ & $1.2 *$ & $0.86 *$ & 0.89* \\
\hline 5 & csiro-mk3-0 & 1.6 & $2.1^{*}$ & $2.1 *$ & $1.4^{*}$ & $1.0^{*}$ \\
\hline 6 & csiro-mk3-5 & 1.0 & $1.5^{*}$ & $1.4^{*}$ & $0.84 *$ & $0.97 *$ \\
\hline 7 & gfdl-cm2-0 & 0.8 & $1.4^{*}$ & $1.3^{*}$ & $0.85 *$ & 0.88* \\
\hline 8 & gfdl-cm2-1 & 1.0 & $1.4^{*}$ & $1.2 *$ & $0.84 *$ & $0.90 *$ \\
\hline 9 & giss-aom & 1.0 & $1.6^{*}$ & $1.8^{*}$ & $1.1^{*}$ & $1.0 *$ \\
\hline 10 & giss-model-e-h & 0.99 & $1.3^{*}$ & $1.6^{*}$ & $1.0 *$ & 0.98* \\
\hline 11 & giss-model-e-r & 1.0 & $1.4^{*}$ & $1.6^{*}$ & $1.0^{*}$ & $1.0^{*}$ \\
\hline 12 & ingv-echam4 & 1.4 & $2.2 *$ & $1.8^{*}$ & $1.1^{*}$ & $1.2 *$ \\
\hline 13 & inmem $3-0$ & 1.2 & $1.9^{*}$ & $1.6^{*}$ & $1.1^{*}$ & $1.1 *$ \\
\hline 14 & ipsl-cm4 & 0.82 & $1.1^{*}$ & $1.3^{*}$ & $0.76 *$ & $1.7^{*}$ \\
\hline 15 & miroc3-2-hires & 0.63 & $1.0 *$ & $1.0 *$ & $0.62 *$ & $0.65^{*}$ \\
\hline 16 & miroc3-2-medres & 0.79 & $1.1^{*}$ & $1.1^{*}$ & $0.74 *$ & 0.76* \\
\hline 17 & miub-echo-g & 0.85 & $1.2^{*}$ & $1.3^{*}$ & $0.77 *$ & $\mathbf{0 . 8 3} *$ \\
\hline 18 & mpi-echam5 & 0.84 & $1.3^{*}$ & $1.2 *$ & $0.75 *$ & $\mathbf{0 . 8 1} *$ \\
\hline 19 & mri-cgcm2-3-2a & 1.0 & $1.7^{*}$ & $2.0 *$ & $1.0 *$ & $1.1 *$ \\
\hline 20 & ncar-ccsm3-0 & 1.0 & $1.4^{*}$ & $1.4 *$ & $0.91 *$ & 0.93* \\
\hline 21 & ncar-pcm1 & 1.2 & $1.9^{*}$ & $2.0^{*}$ & $1.1^{*}$ & $1.2 *$ \\
\hline 22 & ukmo-hadem 3 & 0.77 & $1.2^{*}$ & $1.0 *$ & $0.72 *$ & $0.75 *$ \\
\hline 23 & ukmo-hadgem1 & 0.87 & $1.2^{*}$ & $1.2 *$ & 0.78* & $\mathbf{0 . 8 2} *$ \\
\hline
\end{tabular}

The indices significantly (at $2.5 \%$ level) indifferent from unit scaling are marked in bold and indices significantly (at $2.5 \%$ level) greater than zero are labelled with an asterisk

pattern correlation (Fig. 3c) results illustrate nicely the concerted emergence of the signal in the late twentieth century in all seasons; whereas significant pattern similarity in the early twentieth century is sporadic and limited to individual seasons.

\subsection{Is the recently observed trend consistent with climate change projections?}

We investigate the consistency of the recently observed warming with what models projected as response of climate to GS-forcing, given that the observed warming is further subject to internal variability and influenced by other external forcings. For seasonal and annual areaaverage warming in Fig. 2, we find that all model-derived GS signals (grey bars) lie within the uncertainty bound about the observed change indicating the influence of nonGS variability (red bars). From this we conclude that GS forcing is consistent with the observed warming.
These results are further confirmed when taking the spatial pattern of change into account. Figure 4 displays the regression coefficients and their $95 \%$ confidence interval. The observed change is consistent with GS forcing if the uncertainty range of regression coefficients includes unit scaling. The observed annual area-mean warming is not significantly different from projections as unit scaling of all 23 projections is well within the uncertainty bars (see Table 2), this suggests that the hypothesized forcing, GS, is a plausible explanation of the observed annual area-mean warming, with a probability of error less than $2.5 \%$. In spring, the regression coefficient of the observed change on GS signals from individual models is not significantly (at $2.5 \%$ level) different from unit scaling with 18 out of 23 models (see Fig. 4). In summer the uncertainty ranges on regression coefficients include unity with 14 out of the 23 models. This suggests that in summer and spring some of the models significantly underestimate the amplitude of observed warming. In autumn the uncertainty range of regression indices includes unity in all cases. Thus, we find consistency with all of the 23 models in autumn.

\subsection{Is the observed change a plausible illustration of future expected changes?}

In this section, we analyze whether the observed warming in the Mediterranean is indistinguishable from the range of CMIP3 projections, i.e. whether the CMIP3 projections encompass the observed warming. If this is the case, we conclude that the observed warming serves as a plausible illustration of future change to be expected in this region. When analyzing area-average warming (Fig. 2), we find that the projections encompass the observed warming in all seasons except in summer. Thus we conclude that the observed area-average warming can be used to illustrate the future expected warming in the Mediterranean except in summer.

When taking into account the spatial pattern of the change as well, we find that regression estimates encompass unit scaling in all seasons (see Table 2). However in spring and summer, most of the projections underestimate the observed warming thus resulting in regression coefficients larger than one. These results together with the low centred correlation coefficients point to the fact, that the spatial features of the observed warming do not well represent the expected future warming due to GS forcing.

\section{Discussion and conclusions}

In this study, we determine if the observed trends in near surface temperature over the period from 1980 to 2009 are consistent with the expected change due to GS forcing. To do so, we consider a large number of climate change 
projections according to the SRES A1B scenario, generated by 23 global models included in the CMIP3 database. If the simulated changes are not significantly different from the observed change, we conclude that anthropogenic forcing is a plausible explanation of the observed change. We estimate significance using 1,000 moving blocks bootstrap replicates of the observed record.

Using an observationally based estimate of "non-GS" variability (internal variability plus other unaccounted factors), we can detect externally forced changes in the observed annual area-mean warming and in all seasons except in winter (with a probability of error of less than $5 \%$ ). We conclude that we need GS forcing for reconstructing the recent trends. Furthermore, we find that the observed area-average warming is consistent with the response to GS forcing as derived from the 23 models. The consistency of observed and projected warming in areamean quantities is largely confirmed when looking at spatially explicit pattern correlation statistics. Both with un-centred pattern correlation as well as with regression, we find generally high similarities of the patterns of observed and projected warming, which can hardly be explained as a result of the "non-GS" variability. Instead, the similarity is evidence that the large-scale component (spatial-mean) of GS-forcing has an important and dominant influence on recently observed warming trends. In contrast, we cannot explain the spatial anomalies of the warming patterns with GS forcing. This is either due to the masking of small-scale features of the GS signal by other non-GS variability or due to the fact that the spatial anomaly pattern derived from global climate model simulations is considerably flawed due to the models coarse horizontal resolution.

Some of the models used in this study ( 9 of 23 models in summer and 5 of 23 models in spring), however, do not reproduce the observed amplification of warming. Most likely candidates to explain the observed summer and spring warming amplification include the response to natural forcing and soil-moisture-temperature feedbacks. As shown by Haarsma et al. (2009) drying leads to a decrease in latent heat flux that in turn leads to strong surface warming in summer. Vautard et al. (2007) identify winter and spring precipitation as a good proxy for summer dryness and excess summer warming in southern and central Europe. Observed precipitation in winter and spring has been decreasing over the Mediterranean during recent decades. This strengthens the hypothesis that this observed amplification of spring and summer warming in the Mediterranean is due to soil-moisture-temperature feedbacks.

Acknowledgments A.B. was funded by CIRCE integrated project. The Climate Research Unit has provided the HadCRUT3 dataset. We further acknowledge the modelling groups, the Program for Climate Model Diagnosis and Intercomparision (PCMI) and the WCRPs Working Group on Coupled Modelling (WGCM) for their roles in making available the WCRP CMIP3 multi-model dataset. The office of Sciences, US Department of Energy, provides support of this dataset. We acknowledge the International Detection and Attribution Group (IDAG) and thanks anonymous reviewers for valuable comments on the manuscript.

Open Access This article is distributed under the terms of the Creative Commons Attribution Noncommercial License which permits any noncommercial use, distribution, and reproduction in any medium, provided the original author(s) and source are credited.

\section{References}

Bhend J, von Storch H (2008) Consistency of observed winter precipitation trends in northern Europe with regional climate change projections. Clim Dyn 31:17-28

Bhend J, von Storch H (2009) Is greenhouse gas forcing a plausible explanation for the observed warming in the Baltic Sea catchment area? Boreal Environ Res 14:81-88

Brohan P, Kennedy JJ, Harris I, Tett SFB, Jones PD (2006) Uncertainty estimates in regional and global observed temperature changes: a new data set from 1850. J Geophys Res 111

Christidis N, Stott PA, Zwiers FW, Shiogama H, Nozawa T (2010) Probabilistic estimates of recent changes in temperature: a multiscal attribution analysis. Clim Dyn 34:1139-1156

Haarsma RJF, Hurk BV, Hazeleger W, Wang X (2009) Drier Mediterranean soils due to greenhouse warming bring easterly winds summertime central europe. Geophys Res Lett 36:340

Hegerl GC, Zwiers FW, Braconnot P, Gillett NP, Luo Y, Marengo Orsini JA, Nicholls N, Penner JE, Stott PA (2007) Understanding and attributing climate change. In: Solomon S, Qin D, Manning M, Chen Z, Mariquis M, Averyt K, Tignor M, Miller H (eds) Climate change 2007: the physical science basis. Contribution of working group I to the fourth assessment report of the intergovernmental panel on climate change. Cambridge University Press, Cambridge, pp 663-745

Lionello P, Malannotte-Rizzoli P, Boscolo R (1999) Mediterranean climate variability. Elsevier, Amesterdam, p 415

Meehl GA, Covey C, Delworth T, Latif M, McAvaney B, Mitchell JFB, Stouffer RJ, Taylor KE (2007) The WCRP CMIP3 Multimodel dataset-a new era in climate change research. Bull Am Meteorol Soc 88:1383-1394

Santer BD, Wigley TML, Jones PD (1993) Correlation methods in fingerprint detection studies. Clim Dyn 8:265-276

Stott PA, Stone DA, Allen MR (2004) Human contribution to the European heatwave of 2003. Nature 432:610-614

Stott PA (2003) Attribution of regional-scale temperature changes to anthropogenic and natural causes. Geophys Res Lett 30

Stott PA, Tett SFB (1998) Scale-dependent detection of climate change. J Clim 30:3282-3294

Vautard R et al (2007) Summertime european heat and drought waves induced by wintertime Mediterranean rainfall deficit. Geophys Res Lett 34

von Storch H, Zwiers FW (1999) Statistical analysis in climate research. Cambridge University Press, London, p 528

Wilks DS (1997) Resampling hypothesis tests for autocorrelated fields. J Clim 10:65-82

Zhang XB, Zwiers FW, Stott PA (2006) Multimodel multisignal climate change detection at regional scale. J Clim 19:4294-4307

Zwiers FW, Zhang XB (2003) Toward regional-scale climate change detection. J Clim 16:793-797 